95\%CI:1.76-2.50) compared to being aged $\geq 35$ years. 1,386 men did not receive the vaccine. Of these men, the majority $(55.4 \% ; n=768)$ were not offered the vaccine by their treating clinician, and $21.6 \%(\mathrm{n}=300)$ were identified as nonimmune after serological testing but did not return for vaccination. By the end of 2018 , a total of $85.5 \%$ of MSM $(8,196 / 9,582)$ were immune to hepatitis A.

Conclusion The critical vaccination threshold for hepatitis A has been estimated at $\geq 70 \%$. Continuation of the targeted hepatitis A vaccination program will improve immunity amongst the MSM population to prevent ongoing transmission and the likelihood of future outbreaks.

Disclosure No significant relationships.

\section{P535 CHEMSEX \& AMP; SEXUAL CONSENT: A QUANTITATIVE STUDY ON SEXUALIZED DRUG USE IN MEN WHO HAVE SEX WITH MEN AND NON-CONSENSUAL SEX}

${ }^{1}$ Jilke Speulman, ${ }^{1}$ Susanne Druckler*, ${ }^{1}$ Martijn Van Rooijen* ${ }^{*}{ }^{2}$ Henry De Vries. ${ }^{1}$ Public Health Service of Amsterdam, Infectious Diseases, Amsterdam, Netherlands; ${ }^{2}$ Public Health Service Amsterdam, Amsterdam University Medical Center (UMC), National Institute of Public Health and the Environment (RIVM), Infectious Diseases, Infection and Immunity Institute (Al and II), Epidemiology and Surveillance Unit, Amsterdam, Netherlands

\subsection{6/sextrans-2019-sti.612}

Background Chemsex, the use of drugs (including crystal methamphetamine, mephedrone and/or GHB/GBL) to enhance sex, is practiced by men who have sex with men (MSM) and has risen in the last decade. Non-consensual sex is hypothesized to occur frequently under the influence of chemsex, however has not been investigated much. In this study we aimed to quantify chemsex use in the Amsterdam area and to establish whether non-consensual sex is associated with chemsex engagement.

Methods During the Amsterdam Pride in 2016 and 2018, Amsterdam-located users of a gay dating application were asked about chemsex behavior in the previous 6 months and non-consensual sexual experiences in the past 5 years (the latter in 2018). Non-consensual sex was defined as 'non-consensual sex or experience (e.g. filmed/photographed without consent)'. $\mathrm{X}^{2}$ test for independence was used for statistical analyses.

Results A total of 1833 (2016) and 756 (2018) participants were included of which $28.3 \%$ and $27.8 \%$ engaged in chemsex $(p=0.81)$. In 2018 the occurrence of non-consensual sex was high, but not significantly different among the chemsex $(20.4 \%)$ and non-chemsex $(16.5 \%)$ group $(p=0.22)$. Yet, among those with chemsex being filmed/photographed without consent, taking drugs against ones will and passing out, were reported more often than among those without chemsex $(\mathrm{p}<0.05)$. The intensity of emotional distress related to nonconsensual sex was not significantly different between the chemsex and non-chemsex group $(\mathrm{p}=0.63)$.

Conclusion The proportion of MSM in Amsterdam who engage in chemsex is high but stable over 2016-2018. The proportion of MSM experiencing non-consensual sex is also high, but not associated with chemsex engagement, nor is the intensity of emotional distress related to chemsex. Nonetheless, the type of non-consensual experience differs between the chemsex and the non-chemsex group. Sexual healthcare professionals need to address chemsex use and non-consensual sex during consultations involving MSM and refer men if deemed necessary.

Disclosure No significant relationships.

\section{P538 HIGH HIV INCIDENCE AMONG MEN WHO HAVE SEX WITH MEN IN EIGHT CHINESE CITIES: RESULTS FROM A COHORT STUDY}

${ }^{1}$ Wenting Huang, ${ }^{2}$ Yehua Wang, ${ }^{3}$ Haidong Lu, ${ }^{3}$ Joseph Tucker, ${ }^{3}$ Weiming Tang* ${ }^{1}$ University of North Carolina at Chapel Hill Project-China, Guangzhou, China; ${ }^{2}$ UNC ProjectChina, Guangzhou, China; ${ }^{3}$ UNC Gillings School of Global Public Health, Chapel Hill, USA

\subsection{6/sextrans-2019-sti.613}

Background Knowing the HIV incidence is essential for providing timely intervention among key populations. However, HIV incidence data from men who have sex with men (MSM) in China are limited. In this study, we aim to measure HIV incidence among MSM in eight cities and investigate correlates with incidence.

Methods This study is a secondary analysis of a steppedwedged randomized control trial focusing on promoting HIV testing among Chinese MSM. MSM from eight cities in Guangdong and Shandong Province were recruited and followed from July 2016 to August 2017. Sexual behaviors and HIV testing activity were measured at baseline and during follow-up survey every three months. Participants who reported to have tested HIV at least twice during different follow-up periods were included in this analysis. We defined the seroconversion as a negative HIV testing result followed by a positive result during any of the follow up. We used Cox regression to examine correlates with HIV seroconversion.

Results Of the 1381 participants recruited at baseline, 360 MSM had HIV tests at least twice. At baseline, 87.5\% (315/ $360)$ were never married and $32.8 \%$ (118/360) never disclosed their sexual orientation to anyone other than their sexual partners. During the 12-month follow-up period, $56.67 \%$ (204/360) had multiple male partners, $15.3 \%$ (55/360) had female partners and $14.7 \%$ (53/360) had bisexual behaviors. Overall, 25 men had seroconversion, and the HIV incidence rate was 15.0/100 person-year. The Cox regression model showed higher seroconversion risk was associated with a higher number of female sexual partners $(\mathrm{HR}=3.59$, 95\% $\mathrm{CI}$ : 1.32-9.80). The association between seroconversion and bisexual behavior was marginally significant $(\mathrm{HR}=2.05$, 95\% $\mathrm{CI}$ : 0.98-4.29).

Conclusion HIV incidence is very high among MSM in our study based in China. It was associated with multiple sexual partners, as well as bisexual behaviors. Interventions to prevent HIV transmission, targeting on sexual behavior change are needed.

Disclosure No significant relationships. 